\title{
Ketogenic diet ameliorates lipid dysregulation in type 2 diabetic mice by downregulating hepatic pescadillo 1
}

Jielin Zhou ${ }^{1 \dagger}$, Yao Lu ${ }^{2+}$, Yajing Jia ${ }^{3}$, Jing Lu' ${ }^{1}$ Zhengxuan Jiang ${ }^{4^{*}}$ and Keyang Chen ${ }^{1,3^{*}}$ (1)

\begin{abstract}
Background: Previous reports implied a possible link between PES1 and lipid metabolism. However, the role of PES1 in regulating T2DM related lipid metabolism and the effect of ketogenic diet (KD) on PES1 have not been reported. The aim of present study is to explore the role of PES1 in effects of KD on diabetic mice and its mediated mechanism.

Methods: Male C57BL/6J and KKAy mice were fed with standard diet (SD) and KD, respectively. Simultaneously, McArdle 7777 cells were treated by $\beta$-hydroxybutyric acid ( $\beta-H B)$, Pes 1 siRNA or Pes 1 overexpression plasmid, respectively. Additionally, liver-conditional knockout (CKO) of Pes 1 in vivo was applied.

Results: Hepatic PES1 expression in diabetic mice was markedly increased, which was suppressed by KD feeding with an accompanying reduction of hepatic and plasma triglycerides (TG). In mice with CKO of Pes 1, the protein levels of p300, SREBP1C, FASN, SCD1, Caspase1, NLRP3 and GSDMD were dramatically downregulated in livers, and the plasma and hepatic TG, IL-1 $\beta$ and IL-18 were decreased as well. The similar outcomes were also observed in $\beta-H B$ and Pes 1 knockdown treated hepatocytes. By contrast, Pes 1 overexpression in cultured hepatocytes showed that these levels were significantly enhanced, which were, however reduced under $\beta$-HB treatment. Mechanistically, we discovered that $\beta-\mathrm{HB}$ decreased $\mathrm{CHOP}$ binding to the Pes 1 promoters, resulting in the downregulation of PES1, thereby reducing PES1 binding to p300 and Caspase1 promoters. The inhibition of p300 and Caspase 1 expression elicited the dramatic suppression of acetylation of SREBP1c via its interaction with p300, and the decreased GSDMD levels. Besides, knockdown of Caspase1 also alleviated the TG levels in cultured hepatocytes.
\end{abstract}

Conclusion: KD may improve lipid dysregulation in type 2 diabetic mice by downregulating hepatic PES1 expression.

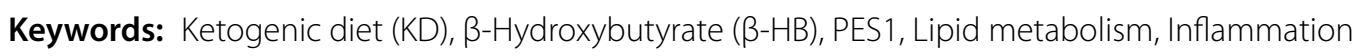

\section{Introduction}

Type 2 diabetes mellitus (T2DM), characterized by insulin resistance (IR) and pancreatic $\beta$-cell dysfunction, is a heterogeneous metabolic disorder (Magliano et al. 2020).

\footnotetext{
*Correspondence: jiangzhengxuan@ahmu.edu.cn; chenkeyang@ahmu.edu. cn

${ }^{\dagger}$ Jielin Zhou and Yao Lu contribute equally to this work

${ }^{1}$ Department of Nutrition and Food Hygiene, School of Public Health, Anhui Medical University, Hefei 230032, Anhui, China

${ }^{4}$ Department of Ophthalmology, The Second Affiliated Hospital, Anhui Medical University, Hefei 230021, Anhui, China

Full list of author information is available at the end of the article
}

Compelling evidence showed that T2DM was accompanied by hypertriglyceridemia-based lipid dysregulation (Zheng et al. 2018; Xu et al. 2018), one of the main factors leading to atherosclerosis and even death (McGuire et al. 2016).

Recently, an interesting dietary pattern, namely ketogenic diet (KD), has been proposed to attain a remarkable decline of hyperglycemia in T2DM (Abbasi 2018). KD is typically characterized by low carbohydrates ( 5 to $10 \%$ of total caloric intake) and high fat consumption (more than $70 \%$ of total caloric intake) (Castellana et al. 2020). Additionally, it was reportedly 
associated with the remission of numerous adverse health outcomes such as obesity, inflammation, cardiovascular disease and cancer (Gentile and Weir 2018; Allen et al. 2014; Yancy et al. 2019; Augustin et al. 2018; Watanabe et al. 2020). One study has documented that the KD could alleviate the hyperglycemia in T2DM through producing ketone bodies that approximately consist of $78 \% \beta$-hydroxybutyric acid $(\beta-\mathrm{HB}), 20 \%$ acetoacetic acid and 2\% acetone (Abbasi 2018; Newman and Verdin 2017). Due to the high fat content in KD, the bad outcomes by the long-term consumption of $\mathrm{KD}$, including no significant weight loss, lipid accumulation, and liver fibrosis, were also reported in some studies (Ellenbroek et al. 2014; Zhang et al. 2016). Nevertheless, the molecular mechanisms underlying the impact of KD have not been satisfactorily explored.

Currently, we unexpectedly observed that hepatic pescadillo 1 (PES1) expression in T2DM mice were markedly elevated. PES1, also known as pescadillo ribosomal biogenesis factor 1, or NOP7, or YPH1, was originally found in zebrafish embryos (Allende et al. 1996). It is evolutionarily highly conserved and forms a complex with BOP1 and WDR12 (PeBoW complex), which is essential for the assembly of $60 \mathrm{~S}$ ribosomal subunits, DNA replication, cell cycle progression, etc. (Du and Stillman 2002; Cheng et al. 2019). PES1 is aberrantly upregulated in various malignant tumors such as breast, ovarian and colon cancers, as well as hepatocellular carcinoma (Cheng et al. 2012; Fan et al. 2018; Li et al. 2013). A considerable number of publications have reported that T2DM with lipid dysregulation is associated with increased incidence and mortality from many cancers (Shlomai et al. 2016; KlilDrori et al. 2017). And one published study discovered that circular antisense non-coding RNA in the INK4 locus (circANRIL), a prototype of circRNA regulating ribosome biogenesis and conferring atheroprotection, acts as a molecular inhibitor of PES1 by binding at the C-terminal domain of PES1 (Holdt et al. 2016). Collectively, these reports suggested the possible link of PES1 to lipid metabolism under T2DM condition.

In this study, we aimed to evaluate the effects of $K D$ on diabetic mice or of $\beta$-HB on liver cells via downregulating PES1 expression, which may be associated with the amelioration of lipid metabolism in vivo and in vitro. Thus, a novel mechanism of PES1 mediated lipid metabolism under T2DM condition affected by KD intervention would be probed. Our current study may provide a novel therapeutic target for T2DM management and treatment by KD or PES1 inhibitor.

\section{Materials and methods \\ Reagents}

$\beta$-HB (166898) was bought from Sigma Chemical Co (St. Louis, MO). Triglycerides (TG), total cholesterol (TC), alanine aminotransferase (ALT), aspartate aminotransferase (AST) and $\beta-\mathrm{HB}$ assay kits were from Nanjing Jiancheng Bioengineering Institute (Jiangsu, China). The ELISA kit for insulin was obtained from Jianglai Corp (Shanghai, China). Flag-tag (20543-1-AP), $\beta$-actin (66009-1-Ig) and Caspase1 (22915-1-AP) antibodies for western bolting, and PES1 (13553-1-AP) antibody for immunofluorescence were obtained from Proteintech, Chicago, USA. PES-1 (NBP2-55211) antibody for western bolting and SREBP1c antibody (NB600-582) for immunofluorescence were bought from Novus Biologicals. SREBP1c (191857), SCD1 (ab19862) and SREBP2 (ab155017) antibodies for western bolting were purchased from Abcam. FASN (3180S) antibody for western bolting was from Cell Signaling Technology (Beverley, MA). NLRP3 (DF7438) and GSDMD (AF4012) antibodies for western bolting were from Affinity. CHOP (sc-7351), p300 (sc-48343) and Ac-SREBP1c (Acetylated-SREBP1c, sc-13551 AC) antibodies were bought from Santa Cruz Biotechnology. RNAex Pro Reagent (AG21102), reverse transcription kits (AG11707), SYBR Green qPCR SuperMix (AG11718) were bought from Accurate Biology. Cell counting kit-8 (CCK-8) (C0005) was from Target Mol (Shanghai, China). Chromatin immunoprecipitation (ChIP) assay kit (P2078) was purchased from Beyotime. Rat Pes1 and Caspase1 short interfering RNA (siRNA) and Pes1 overexpression plasmid were purchased from GENERAL BIOL (Anhui, China).

\section{Animal grouping}

Five-week-old male C57BL/6J and KKA (KK.Cg-Ay/J) mice were from Beijing Vital River. The housing unit was maintained at constant temperature $22-25{ }^{\circ} \mathrm{C}$ and $50-60 \%$ relative humidity with a 12/12-h light/dark cycle and free access to tap water. $K K A^{y}$ mice were fed with commercial high-fat diet (BEIJING HFK Bio-Technology Co., Ltd., Beijing, China) ad libitum for 4 weeks to increase their plasma glucose levels. After 4 weeks, all mice were divided into four groups (10-12 mice per group), including C57BL/6J fed with standard diet (SD, LAD3001G, Trophic Animal Feed High-Tech Co., Ltd, China) (C57BL/6J-SD) and ketogenic diet (KD, TP 201455, Trophic Animal Feed High-Tech Co., Ltd, China) (C57BL/6J-KD), and $\mathrm{KK} A^{\mathrm{y}}$ fed with SD (KK $\left.A^{\mathrm{y}}-\mathrm{SD}\right)$ and $\mathrm{KD}\left(\mathrm{KK} A^{\mathrm{y}}-\mathrm{KD}\right)$. The composition of the diet was listed in Additional file 1: Table S1. All mice were fed for 16 weeks. The food and water intakes were measured three times a week and taken by the average. Body weight and fasting 
plasma glucose (fasted for 6-8 h) were recorded once a week. Feeding efficiency was calculated as body weight gain (mg) per kcal food consumed. Our procedures on mice obeyed the guidelines for humane treatment set by the Association of Laboratory Animal Sciences at Anhui Medical University.

\section{GTT and ITT tests}

Fasting glucose was measured weekly by Roche blood glucose meter. Glucose tolerance test (GTT) and insulin tolerance test (ITT) were performed before the mice were sacrificed, the concentrations of glucose and insulin for intraperitoneal injection were $2 \mathrm{mg} / \mathrm{g}$ body weight and $0.7 \mathrm{mU} / \mathrm{g}$ body weight, respectively. Blood glucose samples were tested at $0,30,60,90,120,150 \mathrm{~min}$ and 0 , $20,40,60,80,100,120 \mathrm{~min}$, respectively. Area-undercurve (AUC) was calculated by the trapezoid rule.

\section{Murine tissue and blood sample collection}

By the end of experiment, all fasted mice were euthanized with $10 \%$ chloral hydrate and then killed by cervical dislocation to obtain tissues and blood samples. Blood was immediately centrifuged at $3000 \mathrm{rpm}$ for $10 \mathrm{~min}$ and the levels of insulin in plasma were detected by enzyme linked immunosorbent assay (ELISA) kits. The homeostatic model assessment of insulin resistance (HOMA-IR) index was calculated using the following formula: fasting insulin levels $(\mathrm{mIU} / \mathrm{L}) \times$ fasting glucose levels $(\mathrm{mmol} / \mathrm{L}) / 22.5$. The remaining plasma was stored at $-80{ }^{\circ} \mathrm{C}$ for later analysis. The liver tissues were rinsed with cold phosphate-buffered saline (PBS). Small portions of liver tissues were fixed in $4 \%$ paraformaldehyde solution for oil red $\mathrm{O}$ and hematoxylin-eosin (H\&E) staining respectively. Then two-third of liver per mice was frozen in liquid nitrogen immediately and kept at $-80{ }^{\circ} \mathrm{C}$ for immunoblotting and biochemical analysis. The remaining livers were preserved in RNAlater for qRT-PCR test. TC, TG, ALT and AST were determined using enzymatic kits in accordance with the manufacturer's instruction.

\section{Cell culture}

McArdle 7777 rat hepatoma cells were obtained from the American Type Culture Collection (Manassas, VA, USA, cat\# CRL-1601). McArdle 7777 cells were cultured in DMEM with high glucose concentration $(25 \mathrm{mmol} / \mathrm{L})$, supplemented with $10 \% \mathrm{FBS}$ and $1 \%$ penicillin/streptomycin in an incubation chamber with $5 \% \mathrm{CO}_{2}$ at $37{ }^{\circ} \mathrm{C}$. The concentration and time of $\beta$-hydroxybutyric acid $(\beta-\mathrm{HB})$ treatment were selected depending on cell counting kit-8 (CCK-8) test. Specifically, McArdle liver cells were seeded in the 96-well plates for $24 \mathrm{~h}$. Then by the $\beta$-HB treatment with a series of concentrations $(0,0.25$,
1, 2, $4 \mathrm{mM})$ and times $(0,12,24,48 \mathrm{~h})$, CCK-8 reagents $(10 \mu \mathrm{L})$ were added into the treated cells. Next, the cells were incubated for $1-4 \mathrm{~h}$ at $37{ }^{\circ} \mathrm{C}$. Simultaneously, the medium was treated in the same way. The absorbance at $450 \mathrm{~nm}$ was detected by the microplate reader. Cellular viability was calculated by the absorbance values, by which the most suitable treatment concentration and time of $\beta$-HB were determined.

\section{Quantitative real-time PCR}

The total RNAs were extracted using RNAex Pro reagent. The genomic DNA was removed using RNase-free DNase. Purified total RNA $(1 \mu \mathrm{g})$ was reverse-transcribed using reverse transcriptase. qRT-PCR was performed with a Roche Light Cycler 480 System with SYBR Green Super Mix using gene-specific primers (Additional file 1: Table S2). The comparative Ct method was used to determine the amount of target normalized to an endogenous reference ( $\beta$-Actin) and relative to a calibrator $\left(2^{-\triangle \triangle C t}\right)$.

\section{Immunoblotting}

McArdle 7777 cells and liver tissues (30 mg/per sample) were homogenized in lysis buffer, respectively. Total lysates $(20 \mu \mathrm{g}$ per well) were separated electrophoretically by $10 \%$ SDS-PAGE and transferred onto PVDF membranes. The membranes were respectively incubated for $24 \mathrm{~h}$ with CHOP, PES1, p300, SREBP1c, SREBP2, FASN, SCD1, NLRP3, Caspase 1, GSDMD antibodies at $4{ }^{\circ} \mathrm{C}$. $\beta$-Actin was used as a loading control. After being washed $8 \mathrm{~min}$ for 4 times with TBST buffer, the membranes were incubated with secondary antibodies for $60 \mathrm{~min}$. The enhanced chemiluminescence reagent was used. The signal was then detected by the digital imaging equipment.

\section{Immunofluorescence}

McArdle 7777 cells were seeded onto 12-well plate containing cell climbing slides for $24 \mathrm{~h}$. After $\beta$-HB treatments, cells were washed thrice with PBS and fixed with $4 \%$ paraformaldehyde for $20 \mathrm{~min}$. Nonspecific binding sites were blocked with 5\% normal bovine serum in PBS. The 12-well plates were incubated with the mixture of primary antibodies including PES1 (1:250) and SREBP1c (1:250) at $4{ }^{\circ} \mathrm{C}$ overnight. After PBS washing, the 12-well plates were incubated for $60 \mathrm{~min}$ with the Alexa Fluor 488 conjugated secondary antibody (ab150077, Abcam) or Alexa Fluor 568 conjugated secondary antibody (ab175473, Abcam). Cell climbing slides were taken out from the 12-well plate and buckled upside down on the 
glass slide containing DAPI. All sections were mounted and observed by a confocal microscope (LSM880, Zeiss).

\section{RNA interference of Pes 1 and Caspase 1}

Rat Pes1 and Caspase1 small interfering RNA (siRNA) and Lipofectamine 3000 (L3000008, Invitrogen Life Technologies Crop) were mixed in serum-free medium for $20 \mathrm{~min}$, respectively. Then the mixture was added to the culture medium to transfect McArdle 7777 cells. After $6 \mathrm{~h}$, the cells continue to be cultured in fresh medium for $48 \mathrm{~h}$. Finally, cells were collected for subsequent measuring TG, TC, and running Western Blotting and qRT-PCR. The sequences of Pes 1 siRNA were 5'-UGAAGAAGCGAGAGAAGUATTT-3' (forward) and 5'-UACUUCUCUCGC UUCUUCATT-3' (reverse). The sequences of Caspase1 siRNA were 5'-AGGAAGA GAUGGAUACAAUTT-3' (forward) and 5'-AUUGUA UCCAUCUCUCUUCCUTT-3' (reverse). The scrambled siRNA control sequences were 5'-UUCUCCGAA CGU GUCACGUTT-3' (forward) and 5'-ACGUGACACGUU CGGAGAATT-3' (reverse).

\section{Overexpression of Pes 1 in vitro}

The purified plasmid of Pes1-flag and Lipofectamine 3000 were mixed in serum-free medium for $20 \mathrm{~min}$. Then the mixture was added into the culture medium to transfect McArdle 7777 cells for $6 \mathrm{~h}$. After being cultured in fresh medium for $24 \mathrm{~h}$, the cells were divided into three groups for different treatments, including negative control (without transfection of Pes1 plasmid or any reagent), Pes1-plasmid and Pes1-plasmid plus $\beta$-HB. After being cultured for $24 \mathrm{~h}$, cells were collected for subsequent assays of TG, TC, Western Blotting and qRT-PCR.

\section{Chromatin immunoprecipitation}

The binding of CHOP (C/EBP-homologous protein) to the Pes1 promoter, PES1 binding to the $p 300$ and Caspase1 promoters were analyzed by chromatin immunoprecipitation (ChIP). Briefly, cells were crosslinked with $1 \%$ formaldehyde for $10 \mathrm{~min}$, neutralized with $125 \mathrm{mM}$ glycine pH 2.5 and washed in PBS. Nuclei were prepared by hypotonic lysis buffer ( $5 \mathrm{mM}$ Pipes $\mathrm{pH} 6.8,85 \mathrm{mM}$ $\mathrm{KCl}, 0.5 \% \mathrm{NP} 40$ ) and centrifugation, and resuspended in SDS lysis buffer, and incubated on ice for $10 \mathrm{~min}$ to be fully lysed. Chromatin was sonicated with bioruptor (Diagenode) to be 200-1000 bp average fragment size and cleared by centrifugation. The anti-CHOP, anti-PES1 antibodies or control rabbit IgG (AC005, ABclonal Technology Co., Ltd) was respectively added into an aliquot of $200 \mu \mathrm{L}$ sonicated lysate, and then $20 \mu \mathrm{L}$ washed protein A/G-agarose beads (sc-2003, Santa Cruz Biotechnology) was added. The mixture was rotated at $4{ }^{\circ} \mathrm{C}$ for $2 \mathrm{~h}$, and then centrifuged at $1000 \mathrm{~g}$ for $1 \mathrm{~min}$ at $4{ }^{\circ} \mathrm{C}$ to wash the beads. The washed beads were resuspended in TE buffer, vortexed $10 \mathrm{~s}$ and boiled for $10 \mathrm{~min}$. The samples and sonicated lysates were treated with $1 \mu \mathrm{L}$ of $20 \mathrm{mg} / \mathrm{mL}$ proteinase $\mathrm{K}$. After being centrifuged at $12,000 g$ for $5 \mathrm{~min}$ at $4{ }^{\circ} \mathrm{C}$, the digested DNA was used for qRT-PCR assay. Primers for the CHOP binding site in Pes1 promoter, the PES1 binding site in the rat $p 300$ and Caspase1 promoters were as follows: CHOP-Pes1 binding site (forward) 5'-CTGGTACGTGGGTGCAGTTTGG-3', CHOP-Pes1 binding site (reverse) 5'-CACACAGGGATGAACATA AGTGAGAGG-3'; PES1-p300 binding site (forward) 5'-TCCTCTTGCTGTCTGACTTGTTTGAG-3', PES1p300 binding site (reverse) $5^{\prime}$-AAGATGTTGAGCCTG TTCTCTGAGTTC-3'; PES1-Caspase1 binding site (forward) 5'-GGAGCAGGGAAACGATGTATGT GAG-3', PES1-Caspase1 binding site (reverse) 5'- TTGCCCTCA GGATCTTGTCTG TTTAAG- ${ }^{\prime}$.

\section{Co-immunoprecipitation}

Every extracted protein stock solution from cultured cells or liver tissues was divided into two aliquots. The small part was directly used for input assay, and the remaining big portion was pre-cleared with protein A/G-agarose (sc-2003, Santa Cruz Biotechnology) for $2 \mathrm{~h}$. The supernatant after pre-clearance was collected by centrifugation, and further divided into two equal portions. Ac-SREBP1c (acetylated SREBP-1c), p300 and nonimmune IgG (Santa Cruz, sc-2025) antibodies were respectively added in the supernatant $(2 \mu \mathrm{g} /$ per portion) and incubated at $4{ }^{\circ} \mathrm{C}$ overnight in rotating equipment. After centrifugation, the precipitates were collected and washed with cold lysis buffer for six times. The mixture of precipitates and loading buffer was boiled and then Ac-SREBP1c, p300, SREBP1c levels were detected using immunoblotting.

\section{Liver-conditional knockout (CKO) Pes 1 gene in mice}

The parent of Pes1 CKO mice were generated by collaboration with Nanjing Institute of Biomedicine, Nanjing University (Jiangsu, China). Positive F1 generation mice were achieved by CRISPR-Cas9 technology. And hepatic Pes1 CKO mice were obtained by positive F1 generation crossing with ALB-Cre mice. Genotyping was performed by PCR using genomic DNA extracted from murine tails at 3-4 weeks. The mice were classified into wild-type littermates (fl/fl, wt/wt) and $\operatorname{Pes}^{(-/-)}(\mathrm{fl} / \mathrm{fl}, \mathrm{mut} / \mathrm{wt})$ relying on genotype. The primer sequences for the transgenic Cre mice were as follows: Cre sense, 5'-TTGGCCCCTTACCATAACTG-3'; Cre antisense, 5'-GAAGCAGAAG CTTAGGAAGATG G-3'. The primer sequences for genotyping the Pes1 alleles were as follows: shared sense, 5'-TTCCTCACC 
CTCAGCATTAG'; wild-type antisense, 5'-GAGATA GACTGCAAGGCACTGT-3'. Only male mice were used for all analyses (10-15 mice per group). After 16 weeks, mice were sacrificed after anesthetization to obtain the serum and livers for consecutive measuring TG, TC, and running Western Blotting and qRT-PCR.

\section{Statistical analysis}

Normally distributed data were expressed as mean \pm SEMs. The continuous variables were analyzed either by Student's $t$ test, or by ANOVA followed by Student-Newman-Keuls q test for multiple comparisons. $P<0.05$ was considered statistically significant. The data were analyzed using SPSS version 22.0 (IBM Corporation, Armonk, USA). Graphics were constructed using GraphPad Prism 7 (GraphPad Software, San Diego, CA).

\section{Results}

Long-term KD intervention improved the hyperglycemia and insulin resistance in diabetic mice

After 16 weeks of KD intervention, murine body weights in $\mathrm{KK} A^{\mathrm{y}}-\mathrm{KD}$ group showed no significant difference from those in the $\mathrm{KK} A^{\mathrm{y}}$-SD groups, the similar results were also observed in the C57BL6J mice (Fig. 1a). With regard to both normal and diabetic mice, intakes of SD were significantly higher than those of KD (Fig. 1b), parallel to the consumptions of water (Fig. 1c). In terms of daily energy intake and feeding efficiency, the values by the KD and SD feeding in normal and diabetic mice were not drastically different (Fig. 1d, e). However, the fasting plasma glucose levels were remarkably reduced in diabetic mice by KD intervention, compared to those by SD (Fig. 1f).

The ITT and GTT assays exhibited that KD could significantly ameliorate glucose tolerance and insulin sensitivity in diabetic mice (Fig. 1g, h). Moreover, serum insulin levels and HOMA-IR in diabetic mice were obviously improved by KD treatments (Fig. 1j, k), but no statistic difference between C57BL/6J-SD and C57BL/6J-KD mice was observed. In addition, the serum $\beta$-HB levels were sharply elevated by KD feeding in both healthy and diabetic mice (Fig. 1i).

\section{KD suppressed PES1 and improved lipid dysregulation under diabetic state}

After 16 weeks of KD feeding, the current results displayed that the protein levels of CHOP, PES1, p300, SREBP1c, N'-SREBP1c, FASN and SCD1 were dramatically inhibited in both healthy and diabetic mice (Fig. 2a, b). Histological analysis (H\&E and Oil Red O staining) revealed much less pathogenesis and lipid accumulation in livers of $K K A^{y}$ mice fed by KD than those by SD (Fig. 2c). Additionally, KD extraordinarily decreased plasma and hepatic TG levels in C57BL/6J and $K K A^{\mathrm{y}}$ mice, compared with SD (Fig. 2d, e). Lastly, KD feeding in normal and diabetic mice was associated with reductions of plasma AST and ALT (Fig. 2f).

\section{$\beta$-HB decreased PES1 and triglycerides in liver cells}

$\beta-\mathrm{HB}$, the main component of ketone bodies, was used to test the KD effect on liver cells in vitro. The optimized concentration and time of $\beta$-HB treatment were selected by cell viability test (Additional file 1: Fig. S1a). The protein levels of PES1 by the $\beta$-HB treatments with different concentrations and times were dramatically lower than those by control treatment (Additional file 1: Fig. S1b, c). Considering the ketogenesis by the KD feeding in mice, the concentration $(1 \mathrm{mM})$ of $\beta-\mathrm{HB}$ and its treatment time $(24 \mathrm{~h})$ were identified for liver cell treatment in vitro, by which the protein levels of CHOP, PES1, p300, SREBP1c, N'-SREBP1c, FASN and SCD1 were greatly attenuated (Fig. 3a, b). In addition, ChIP test indicated that $\beta$-HB decreased CHOP binding to Pes1 promoter (Fig. 3c). Consistent with the above data, the immunofluorescence showed that $\beta$-HB suppressed PES1 and SREBP1c proteins in nucleus of McArdle cells (Fig. 3d). Moreover,

\footnotetext{
(See figure on next page.)

Fig. 1 Ketogenic diet decreased the hyperglycemia and ameliorated insulin resistance in diabetic mice. a Shown are the changes of body weights exerted by the different food feedings in normal and diabetic mice. $\mathbf{b}$ Displayed are the food intakes in different groups throughout 16 weeks of feeding. c Exhibited are the water intakes in different groups throughout 16 weeks. $\mathbf{d}$ Demonstrated are the total energy intakes calculated by calories in different groups throughout 16 weeks. e Unveiled are the feeding efficiencies in different groups (calculated as body weight gain (mg) per kcal food consumed). f Shown are the variations of fasting plasma glucose in different groups throughout 16 weeks. $\mathbf{g}$ Intraperitoneal glucose tolerance tests were performed in different groups by the end of food feeding. $\mathbf{h}$ Intraperitoneal insulin tolerance tests were carried out in different groups by the end of food feeding. Glucose $(2 \mathrm{mg} / \mathrm{g})$ or insulin $(0.75 \mathrm{mU} / \mathrm{g})$ was injected intraperitoneally after mice were fasted for $6-8 \mathrm{~h}$. Blood glucose samples were collected at different indicated time points and measured. Areas under the curve were calculated for the quantitative analysis. $\mathbf{i}$ Demonstrated are the levels of plasma $\beta$-hydroxybutyric acid ( $\beta-H B$ ) in different groups. $\mathbf{j}$ Insulin levels were detected in different groups by the end of food feeding. $\mathbf{k}$ HOMA-IR in different groups was calculated by the formula. Data were shown as mean $\pm S E M$ for each experiment performed independently 3 times $\left(n=10-12\right.$ per group). SD (Standard diet), KD (Ketogenic diet). ${ }^{*} P<0.05$ C57BL/6J-KD vs C57BL/6J-SD, \# $P<0.05$ KKA'-KD vs KKAY-SD (ANOVA, Student-Newman-Keuls q test)
} 
a

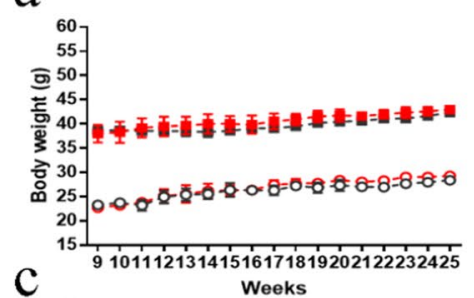

C
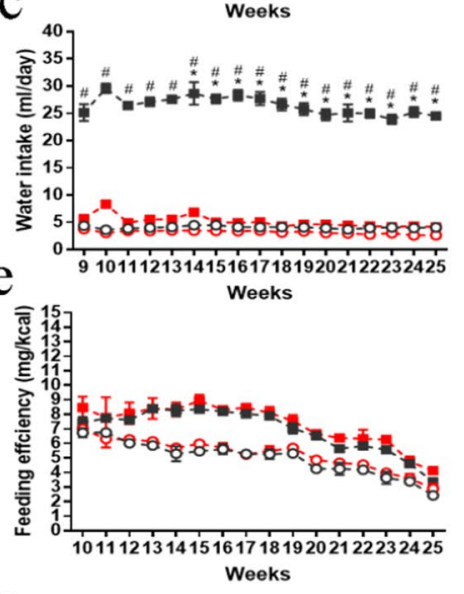

g

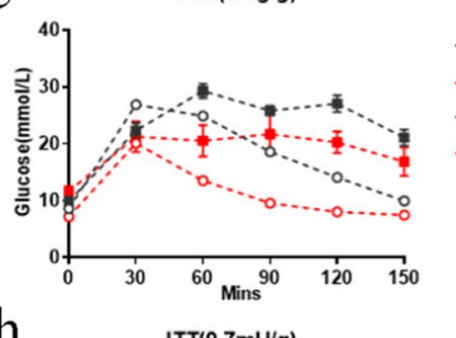

h

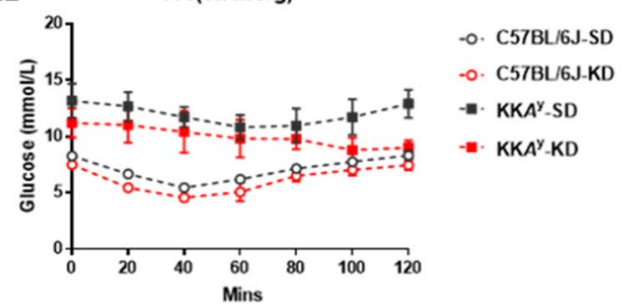

i

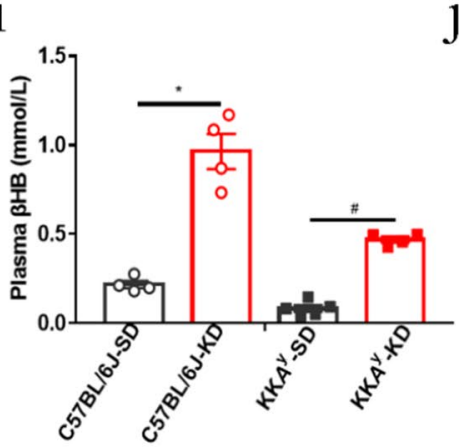

-. C57BL6J-SD

-.. C57BL6J-KD

- $K K A^{\mathrm{y}}$-SD

- $K K A^{\mathrm{y}}-\mathrm{KD}$

j b

-०. C57BL/6J-SD

-०. C57BL/6J-KD

-m. KKA $A^{\mathrm{y}}-\mathrm{SD}$

- $K K A^{y}-K D$

-o. C57BL/6J-SD

-०. C57BL/6J-KD

-m. KKA $A^{\mathrm{y}}-\mathrm{SD}$

-m. KKA $A^{y}-K D$

-o. C57BL/6J-SD

-o. C57BL/6J-KD

-. $\cdot K K A^{\mathrm{y}}-\mathrm{SD}$

-. $K K A^{\mathrm{y}}-\mathrm{KD}$

d

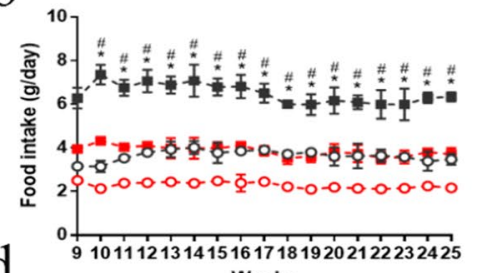

-०. C57BL/6J-SD

-o. C57BL/6J-KD

-m. KKA ${ }^{\mathrm{y}}$-SD

-. $K K A^{\mathrm{y}}-\mathrm{KD}$

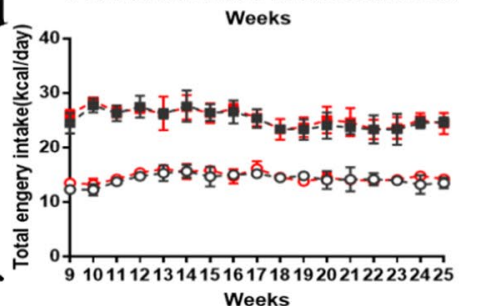

-o. C57BL/6J-SD

-o. C57BL/6J-KD

-m. $K K A^{\mathrm{y}}-\mathrm{SD}$

-m. $K K A^{\mathrm{y}}-\mathrm{KD}$

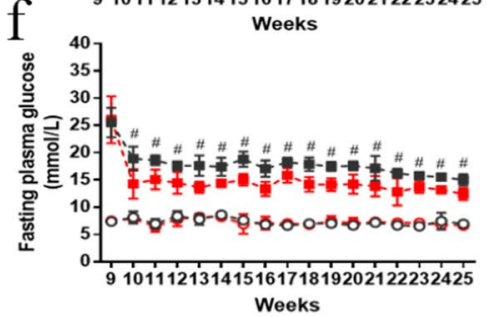

-. C C57BL/6J-SD

-०. C57BL/6J-KD

-. $K K A^{\mathrm{y}}-\mathrm{SD}$

-1. $K K A^{y}-K D$
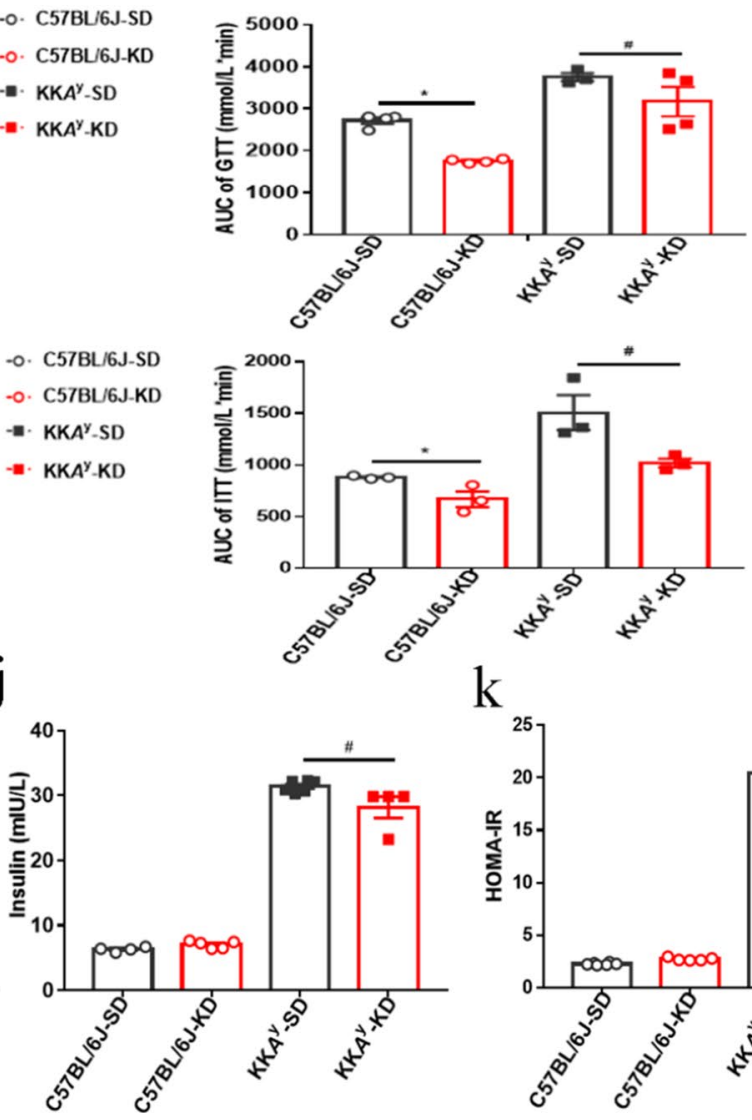

$\mathrm{k}$

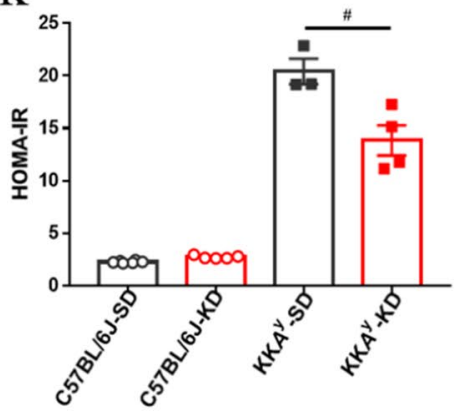

Fig. 1 (See legend on previous page.) 


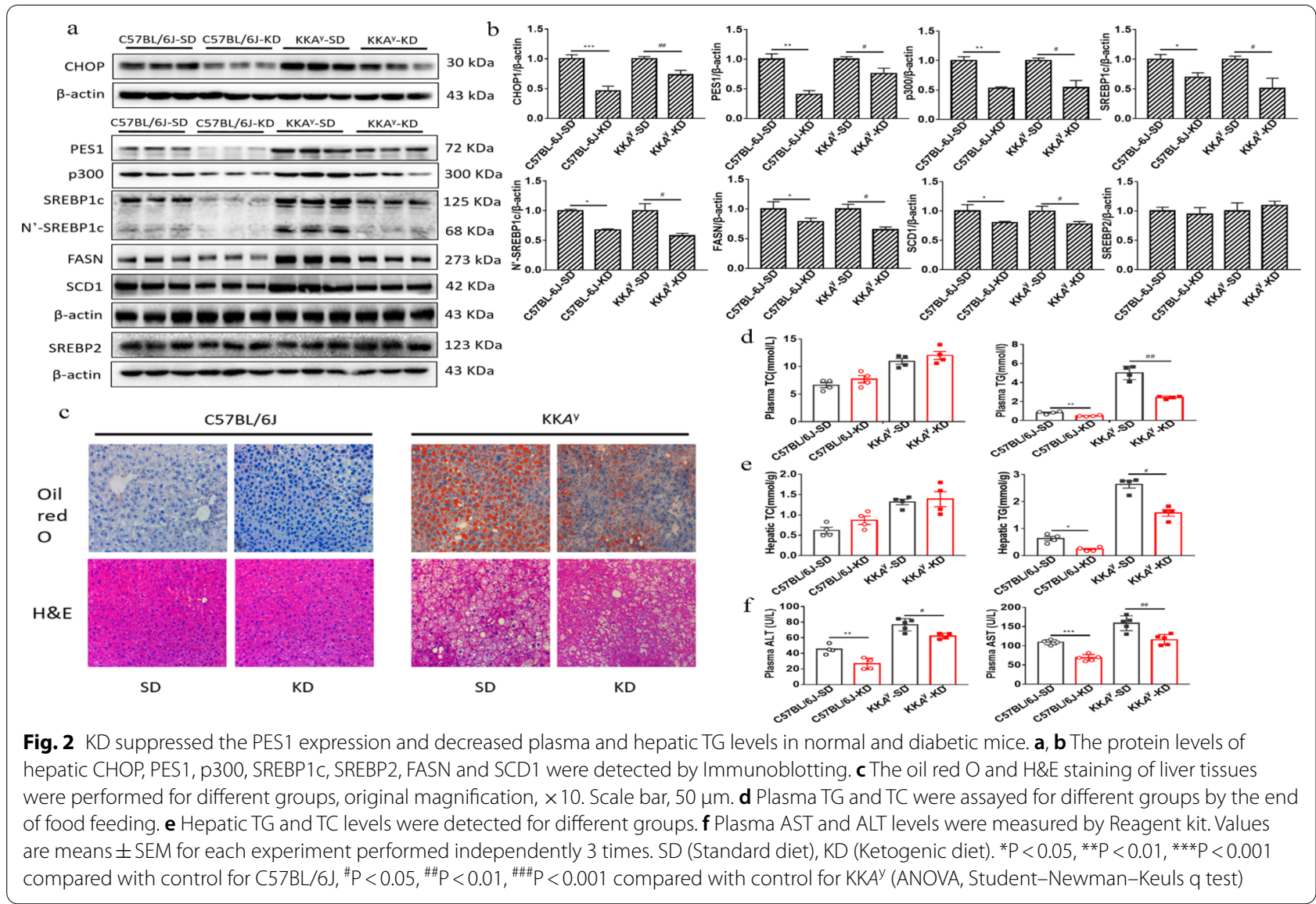

medium and cellular TG levels were markedly declined by $\beta$-HB treatment (Fig. 3e, f).

\section{In vitro knockdown of Pes 1 impaired medium and cellular triglyceride production in liver cells}

To probe PES1 modulating role, the siRNA knockdown of Pes1 was performed in McArdle cells. The levels of PES1 protein were significantly downregulated in the treated cells (Fig. 4a, b). Simultaneously, the medium and cellular triglycerides, and the levels of p300, SREBP1c, N'-SREBP1c, FASN and SCD1 in the treated cells were dramatically reduced compared with those in control cells (Fig. 4a-d).

\section{In vitro supplementation of Pes 1 promoted cellular triglyceride levels, but $\beta-\mathrm{HB}$ treatment eliminated the elevation}

The overexpression of Pes 1 in vitro was performed to further confirm the key role of PES1 in lipid metabolism. The current results showed that the protein levels of p300, SREBP1c, N'-SREBP1c, FASN and SCD1 were significantly increased by Pes1 overexpression, but the effects were sharply reversed by $\beta-\mathrm{HB}$ treatment (Fig. 4e, f). Furthermore, overexpression of Pes1 significantly increased medium and cellular triglycerides, while $\beta-\mathrm{HB}$ treatment eliminated those increases (Fig. 4g, h).

\section{Liver-specific Pes 1 knockout in mice decreased the lipid generation}

The exon 2 in murine Pes 1 gene was targeted by CRISPRCas9/RNA system gene targeting technology (Fig. 5a). The liver mRNA levels of Pes1 were almost completely eliminated in CKO mice by qRT-PCR test (Additional file 1: Fig. S2). After 16 weeks of feeding, no significant difference of body weights between wild-type littermates (fl/fl, wt/wt) and Pes1-CKO mice (fl/fl, mut/wt) was found (Fig. 5b). But the protein levels of p300, SREBP1c, N'-SREBP1c, FASN and SCD1 were dramatically downregulated in Pes1-CKO mice (Fig. 5c, d). Moreover, the weights of liver and subcutaneous fat, and the ratio of liver or fat to body weight were also substantially declined in Pes1-CKO mice (Fig. 5e-g). Likewise, plasma and hepatic triglycerides, plasma AST and ALT in Pes1CKO mice were sharply lower than those in the wild-type littermates (Fig. 5h-j). 

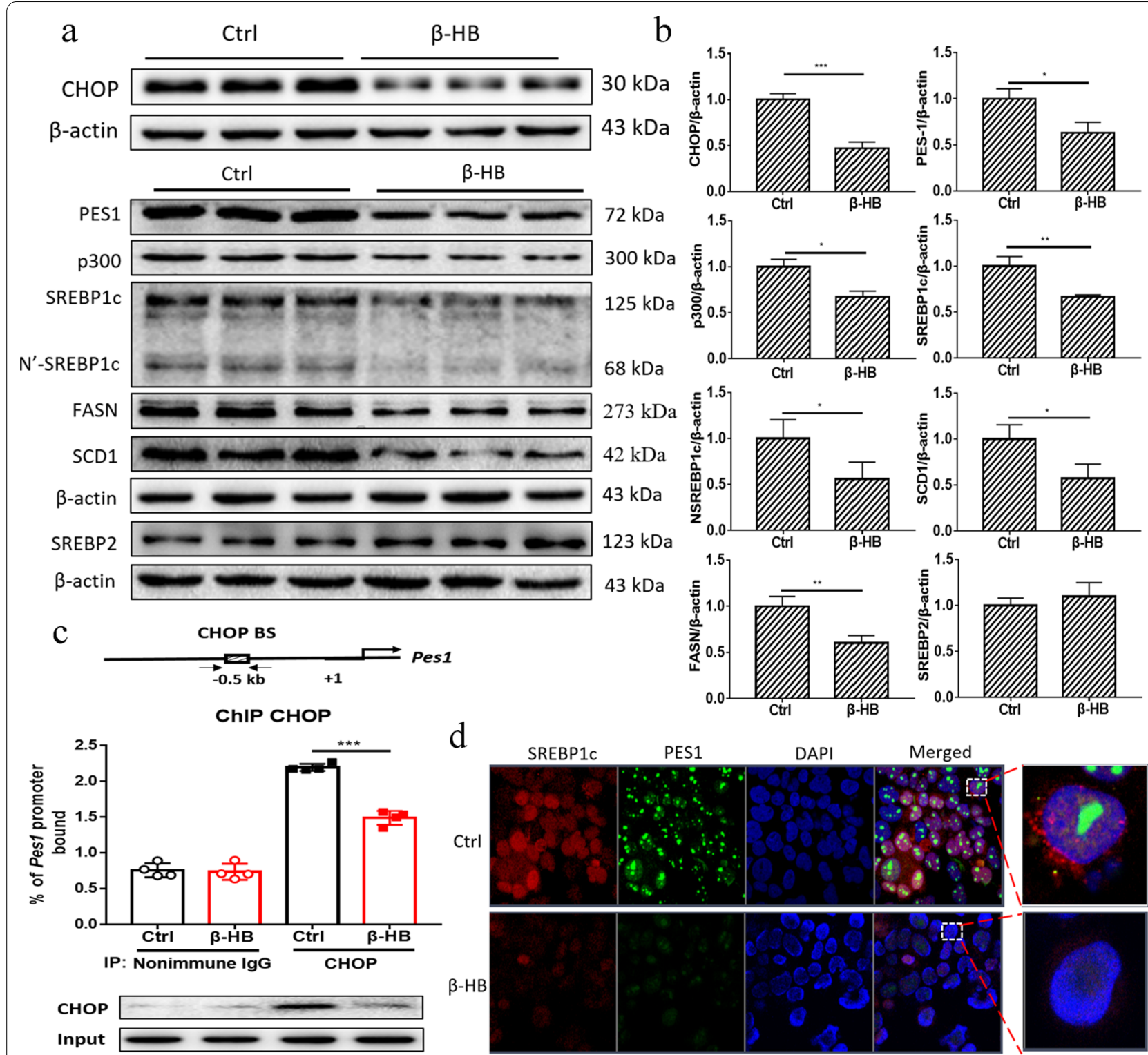

$\beta-\mathrm{HB}$

e

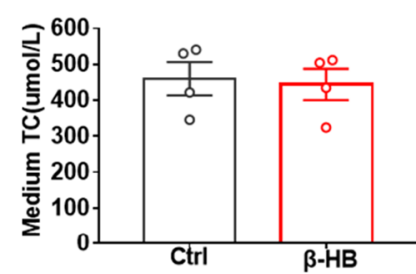

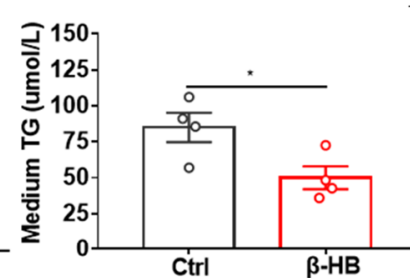

$\mathrm{f}$

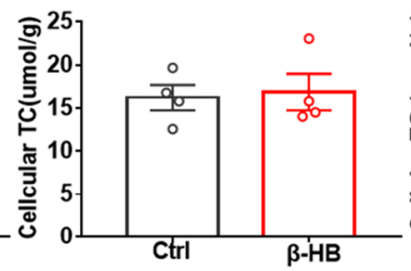

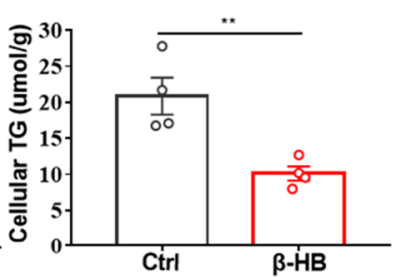

Fig. $3 \beta$-HB decreased PES1 and triglycerides in cultured liver cells. $\mathbf{a}$, b The protein levels of CHOP, PES1, p300, SREBP1C, SREBP2, FASN and SCD1 in hepatocytes were detected by immunoblotting after $1 \mathrm{mM} \beta$-HB treatment for $24 \mathrm{~h}$. c $\beta$-HB decreased CHOP binding to Pes 1 promoter. $\mathbf{d}$ Exhibited are immunofluorescence images of $\beta$-HB-treated liver cells for PES1 and SREBP1c expression and localization, Scale bar represents 20 um. The nuclei were stained with DAPI. e, $\mathbf{f}$ Demonstrated are the TG and TC levels respectively detected in the cultured media and liver cells after $\beta$-HB treatment. $\mathrm{Ctrl}\left(\right.$ Control), $\beta-\mathrm{HB}$ ( $\beta$-hydroxybutyric acid). Data were shown as mean \pm SEM for each experiment performed independently 3 times. ${ }^{*} P<0.05$, ${ }^{*} \mathrm{P}<0.01,{ }^{* *} \mathrm{P}<0.001$ compared with control (Student's t test) 


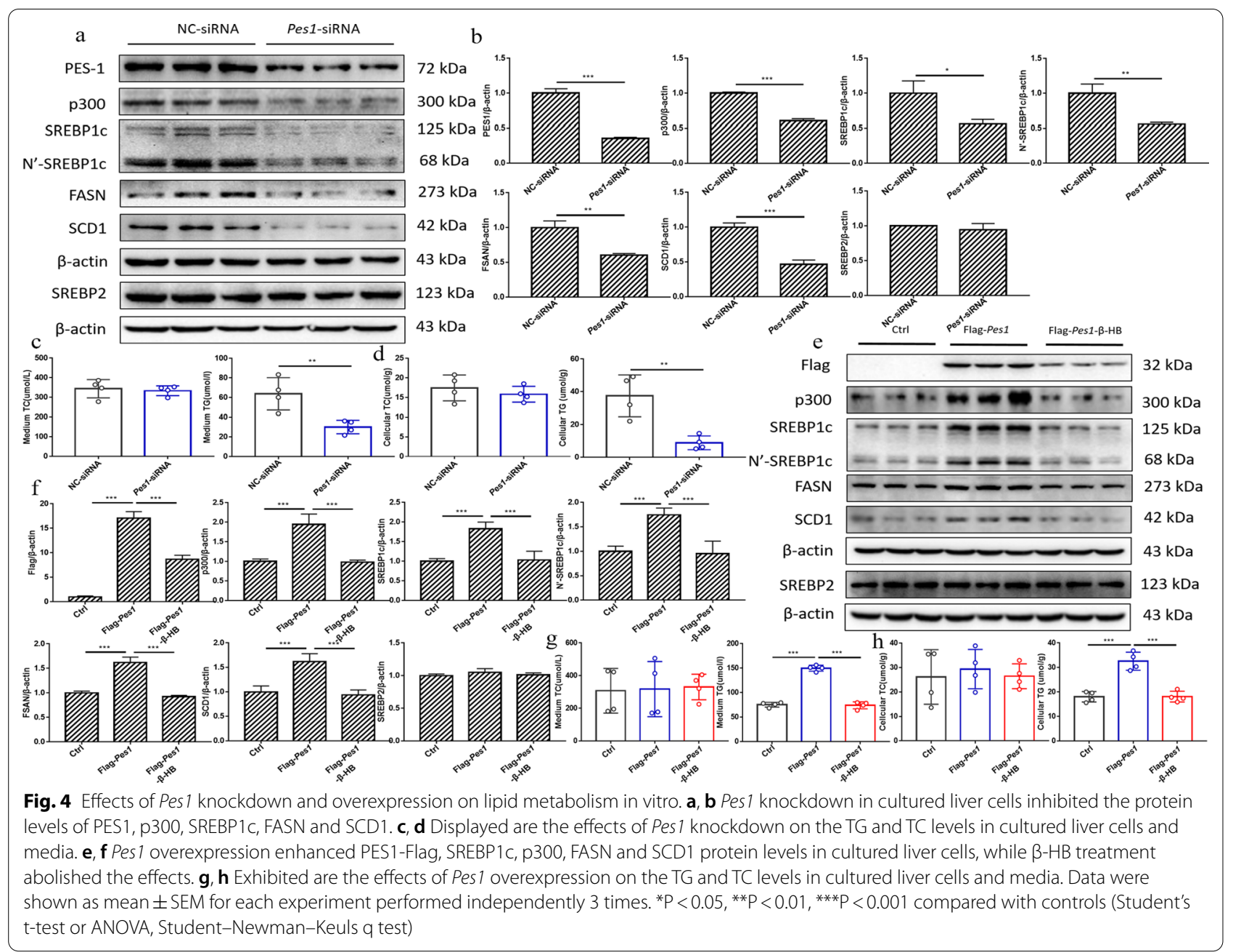

\section{$\beta$-HB lowered PES1 binding to the $p 300$ promoter} and downregulated PES1 mediated acetylation of SREBP1c The ChIP experiment revealed that $\beta$-HB reduced PES1 binding to the $p 300$ promoter, resulting in downregulation of $p 300$ gene expression (Fig. 6a). To determine whether $\beta$-HB affects PES1 regulated SREBP1c activity, acetylation assays both in vitro and in vivo were performed. Additionally, co-IP was used to explore the interaction between p300 and SREBP1c. We found that Pes 1 silence in hepatocytes reduced the interaction between p300 and SREBP1c (Fig. 6b). Contrastingly, Pes1 overexpression increased the interaction, which was however impaired under $\beta$-HB treatment (Fig. 6c). Subsequently, $\beta$-HB treatment in hepatocytes inhibited the acetylation of SREBP1c (Fig. 6d). Next, whether the acetylation of SREBP1c is regulated by PES1 in vivo was also investigated. Our current data indicated that Pes1 knockout in mice significantly reduced the interaction between p300 and SREBP1c (Fig. 6e). And KD feeding in normal and diabetic mice showed that the acetylation of SREBP1c was sharply suppressed (Fig. 6f).

\section{$K D$ and $\beta$-HB decreased inflammation responses by downregulating PES1}

According to the published reports, inflammation might promote lipogenesis (Todoric et al. 2020). In the present study, the levels of Recombinant NLR Family Pyrin Domain Containing Protein 3 (NLRP3), Caspase1, Cleaved-Caspase1, gasdermin-D (GSDMD), and Cleaved-GSDMD were found to be significantly inhibited in normal and diabetic mice fed with KD, compared with those fed with SD (Fig. 7a, b). Simultaneously, KD led to much less inflammation responses in mice than did SD, as indicated by decreased pro-inflammatory factor levels ( $I L-1 \beta$ and $I L-18)$ in the murine livers (Fig. 7c), similar to the results of $\beta$-HB treated hepatocytes (Fig. $7 d-f$ ). Furthermore, the silence or supplementation of Pes1 in vitro 


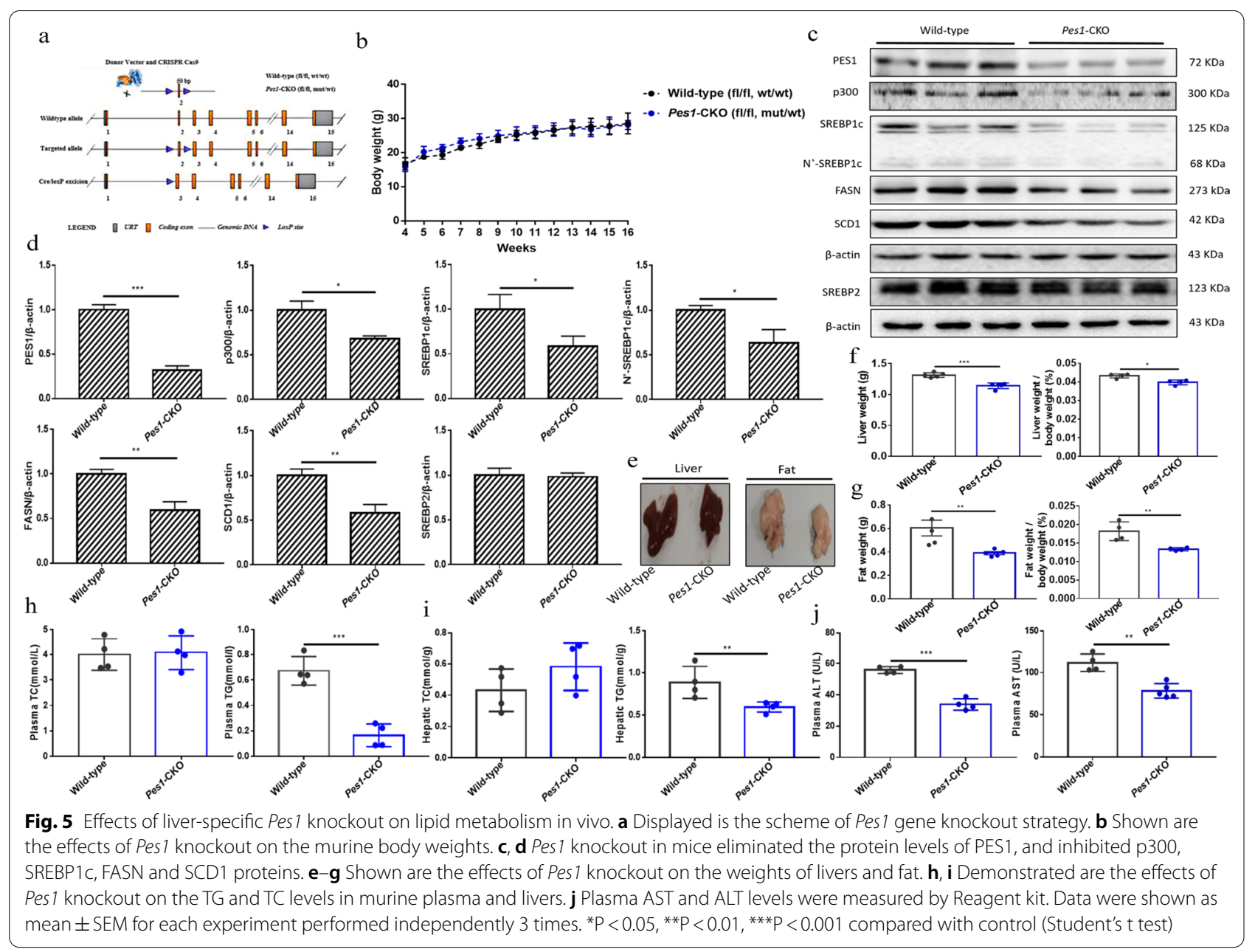

elicited the suppression or enhancement of NLRP3, Caspase 1, Cleaved-Caspase 1, GSDMD, Cleaved-GSDMD, $I L-1 \beta$ and $I L-18$ levels, respectively (Fig. $7 \mathrm{~g}-\mathrm{i}$ ). More interestingly, the enhancement by overexpression of Pes 1 was also impaired with $\beta$-HB treatment in vitro (Fig. 7j1). In addition, consistent with Pes1 silence in vitro, the similar results were observed in Pes1 knockout mice (Fig. $7 \mathrm{~m}-\mathrm{o}$ ).

\section{$\beta$-HB decreased PES1 binding to the Caspase1 promoter and knockdown of Caspase1 reduced medium and cellular triglycerides in liver cells}

To verify Caspase1 modulating role, the siRNA knockdown of Caspase1 was carried out in hepatocytes. The knockdown results suggested that the levels of Caspase1 and GSDMD were declined (Fig. 8a, b), and that the mRNA levels of $I L-1 \beta$ and $I L-18$ were suppressed as well (Fig. 8c). Furthermore, medium and cellular TG levels were dramatically inhibited in Caspase1 siRNA treated cells (Fig. 8d, e). Mechanistically, $\beta$-HB treatment in liver cells lowered PES1 binding to Caspase1 gene promoter, resulting in the downregulation of Caspase 1 gene expression (Fig. 8f). The schematic diagram of entire study is presented in the Fig. 8g.

\section{Discussion}

Recently, clinical studies indicated that KD was effective in improving metabolic parameters of weight, glycemia, and lipid profiles in patients with overweight or obesity, especially in those with preexisting diabetes (Choi et al. 2020; Li and Heber 2020). In our current study, although the high glucose level is naturally dropped in $\mathrm{KK} A^{\mathrm{y}}$ murine model after age of 18 weeks according to the literature (Zhang et al. 2020), we still observed the effects of $\mathrm{KD}$ on lowering plasma glucose and improving insulin sensitivity in T2DM mice compared with those of SD, which was consistent with previous publication (Yang et al. 2021). In addition, Guo et al. (2020) also reported that the plasma and hepatic TG levels were evidently dropped in normal and T2DM mice fed by KD, which are exactly resembling to our current study. 


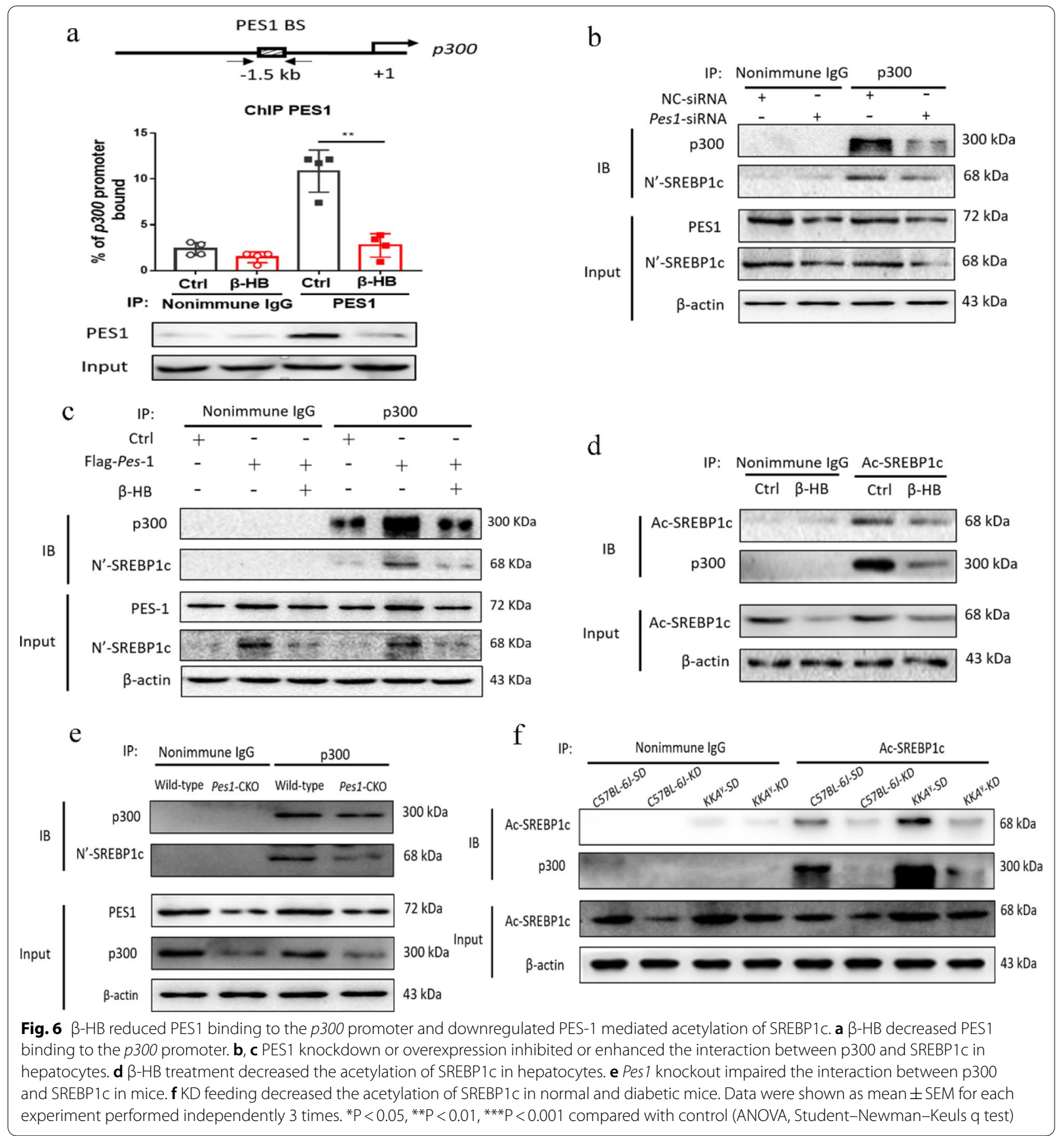

$\beta-\mathrm{HB}$, as the predominant component of ketone bodies, has been demonstrated to serve as molecular scaffold that regulates cellular function by directly activating hydroxy-carboxylic acid receptors, and to be involved in multiple physiological processes including metabolism and inflammation (Offermanns 2017. In this study, the levels of $\beta$-HB in normal mice were higher than those in diabetic mice after KD treatment. This may be because the ketone bodies are produced by fatty acid $\beta$-oxidation, which is upregulated by carnitine palmitoyltransferase 1 (CPT1) (Puchalska and Crawford 2017). Similarly, 3-hydroxymethylglutaryl-CoA synthase 2 (HMGCS2) is a rate-limiting enzyme that promotes $\beta$-HB synthesis as well (Shimazu et al. 2010). In our current work, 


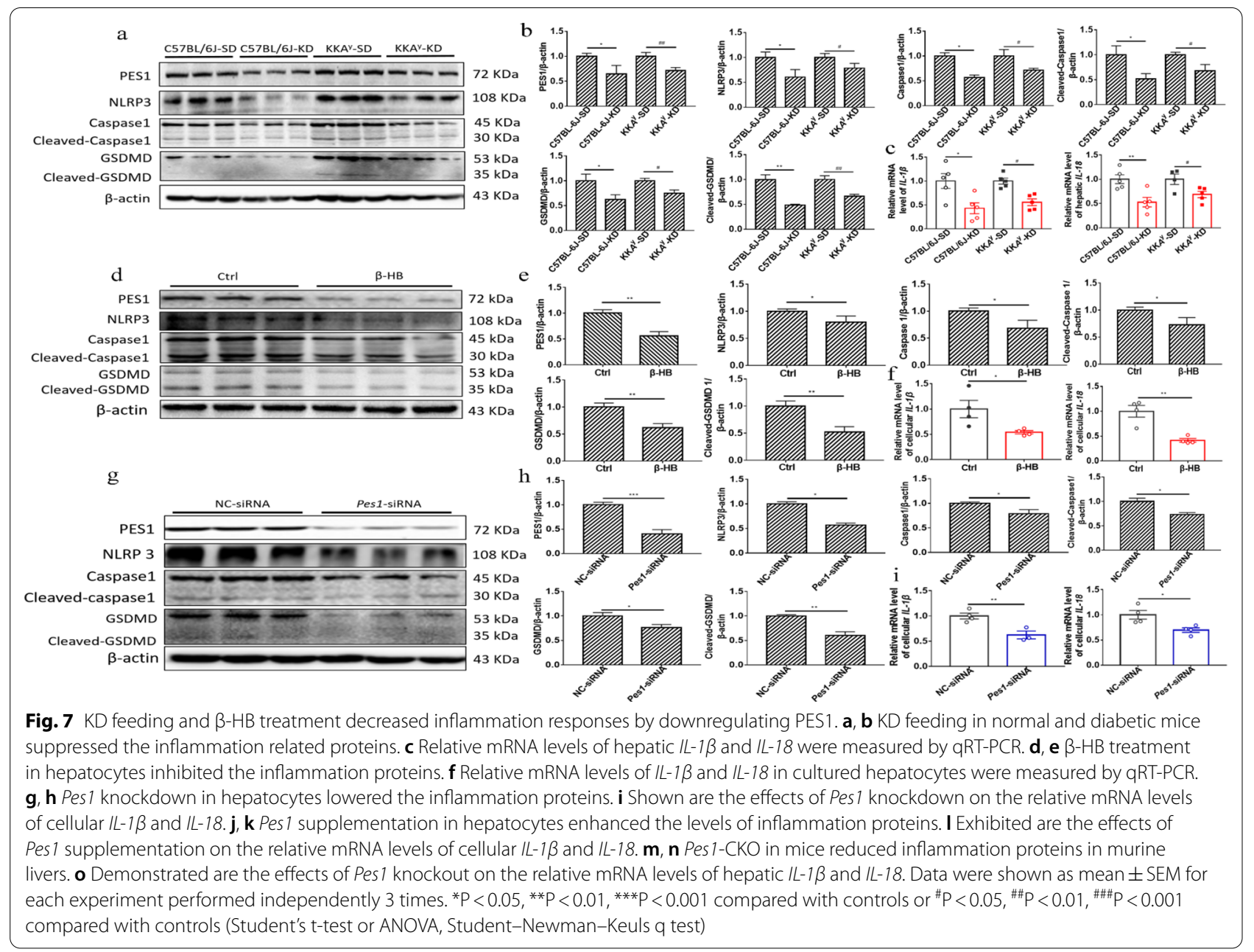

although the expression of CPT1 and HMGCS2 by KD treatment is higher than that by SD in both $\mathrm{C} 57 \mathrm{BL} / 6 \mathrm{~J}$ and $\mathrm{KK} A^{\mathrm{y}}$ mice, the latter ones fed with SD or KD had much less expression of CPT1 and HMGCS2 than the previous ones (Additional file 1: Fig. S3). These results suggested that the $\beta-\mathrm{HB}$ levels in $\mathrm{KK} A^{\mathrm{y}}$ mice may be lower than those in C57BL/6J mice after 16-week treatment with the same KD, as shown in our Fig. 1i. In addition, emerging evidence from long-term KD intervention in humans supports that the ketone level was also attenuated in T2DM patients (Landry et al. 2021). And the initial $\beta-\mathrm{HB}$ value was below ketosis level at the beginning of the KD treatment $(0.12 \mathrm{mmol} / \mathrm{L})$, then peaked at $1.1 \mathrm{mmol} / \mathrm{L}$ (4 weeks) and decreased to $0.51 \mathrm{mmol} / \mathrm{L}$ by week 12 in the end (Landry et al. 2021). Moreover, $\beta$-HB levels are significantly different among different mice by $\mathrm{KD}$ intervention based on previous reports. For example, the $\beta$-HB levels in $d b / d b$ mice were dramatically higher than those in wild type mice after KD intervention, while the enhanced degree of $\beta$-HB levels in $o b / o b$ mice relative to their controls fed with the same KD were much lower than that in $d b / d b$ mice (Thio et al. 2006; Poplawski et al. 2011; Badman et al. 2009). Furthermore, our current data indicated that the elevation fold of $\beta$-HB level in $K K A^{y}$ mice was even moderately higher than that in C57BL/6 mice comparing KD feeding to SD, but no significant difference between two ratios was observed (Additional file 1: Fig. S4). Additionally, the fasting plasma glucose, HOMA-IR and TG levels were markedly reduced in KDfed $K K A^{\mathrm{y}}$ mice compared with SD-fed controls, suggesting the effective intervention by KD-derived $\beta$-HB levels in vivo. Taken together, although the ketogenesis by $\mathrm{KD}$ was seemingly mitigated in $\mathrm{KK} A^{\mathrm{y}}$ mice compared with that in C57BL/6J mice, the ketone level was still legitimate for effectiveness in $\mathrm{KK} A^{\mathrm{y}}$ mice in our current study based on the preceding findings and present results.

Of note, we discerned that PES1 levels were downregulated by $\mathrm{KD}$ feeding in normal and diabetic mice and by $\beta-\mathrm{HB}$ treatment in cells. Previous finding indicated that circANRIL could play an important role in 

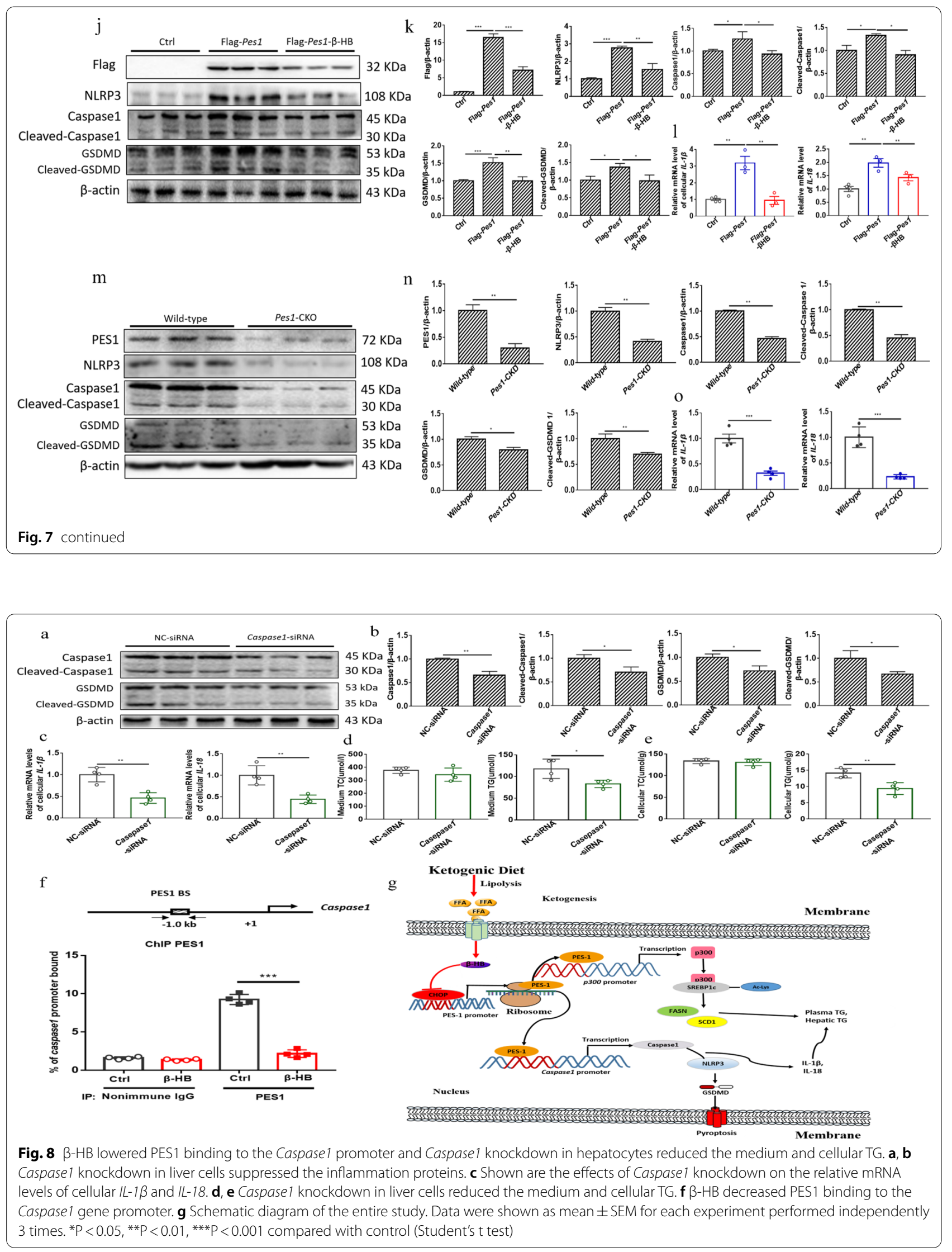
atheroprotection by inhibiting PES1 (Holdt et al. 2016). Moreover, our current study unveiled that $\beta$-HB may reduce $\mathrm{CHOP}$ binding to Pes1 promoter, resulting in the downregulation of PES1. The decreased PES1 protein level impaired its binding to $p 300$ and Caspase 1 promoters, causing the declined expressions of p300 and Caspase1, thus undermining p300 mediated acetylation of SREBP1c and inflammation response. CHOP, a key apoptosis-promoting factor, tightly controls the gene transcription and has pivotal roles in lipid accumulation, adipogenesis and inflammatory action (Liu et al. 2018; Maris et al. 2012; Allagnat et al. 2012). And one report has indicated that KD intervention in mice could reduce the expression of CHOP (Yu et al. 2020), which is consistent to our current study. In addition, it has also been proposed that SREBP1c binds to its lipogenic target genes, such as fatty acid synthase (FASN) and stearoyl-CoA desaturase 1 (SCD1), thereby stimulating de novo lipogenesis ( $\mathrm{Li}$ et al. 2015). A crucial role of SREBP1c has been shown in vivo by hepatic overexpression of SREBP1c in transgenic mice that led to increased hepatic lipid accumulation (Wang et al. 2015). Accumulating evidence showed that the acetylation of SREBP1c increased its stability and activity, and SREBP1c is acetylated by p300 at Lys-289 and Lys-309 (Ponugoti et al. 2010). In this report, after Pes1 gene was knocked down, the interaction between SREBP1c and p300 was significantly inhibited, consistent with our observation in Pes1-CKO mice. Moreover, the interaction between SREBP1c and p300 was obviously strengthened during Pes1 gene overexpression, which was however impeded by $\beta-\mathrm{HB}$ treatment. Furthermore, our current study also suggested that $\beta-\mathrm{HB}$ and KD remarkably reduced the SREBP1c activity via inhibiting the acetylation of SREBP1c.

Compelling evidence based on animal study suggested that KD may block NLRP3 inflammasome-mediated IL-1 $\beta$ and IL-18 production (Youm et al. 2015), which is in concordance with our current observation. In addition, after in vitro knockdown of Pes1 and in vivo ablation of Pes1, the expression of NLRP3, Caspase1 and GSDMD was significantly inhibited. This is opposite to that after overexpression of Pes 1 in vitro. However, the Pes1 overexpression induced effect was abolished by $\beta-\mathrm{HB}$ treatment. These results suggested that $\beta-\mathrm{HB}$ plays a vital role in regulation of inflammation response by downregulating PES1. Moreover, Caspase1 and NLRP3, as regulatory molecules of lipid metabolism, were reported to promote the release of mature IL- $1 \beta$ and to interfere insulin signaling and production, subsequently leading to the lipogenesis and fat accumulation (Anand 2020). Based on Caspase1 knockdown in hepatocytes, our current data exhibit that medium and cellular TG levels are clearly curbed, which is similar to the protection role in Caspase1 knockout mice fed with high fat diet (Stienstra et al. 2011). Collectively, these results suggest that both $\beta$-HB treatment in cells and KD feeding in mice greatly reduced lipid production or accumulation via downregulating PES1 modulated acetylation of SREBP1c and inflammation pathways.

The current study explored a novel mechanism of KD intervention in diabetic mice, which may have translational potential and clinical implications by finding a PES1 inhibitor. However, the interpretation of this study so far is still limited due to the lack of human intervention data, and PES1 function and its mediated mechanism by KD intervention in human is needed in future. In addition, the metabolic factors regulating PES1 expression under T2DM condition are not elucidated yet.

\section{Conclusion}

In summary, our current findings demonstrate that $\mathrm{KD}$ may ameliorate lipid deposition in type 2 diabetic mice by downregulating PES1 modulated acetylation of SREBP1c and inflammation pathways. This study may provide a novel insight to understanding the mechanisms underlying T2DM related lipid dysregulation and contribute a new pharmaceutical target for T2DM treatment.

\section{Abbreviations \\ ALT: Alanine aminotransferase; AST: Aspartate aminotransferase; AUC: Area- under curve; CCK-8: Cell counting kit-8; CHOP: C/EBP-homologous protein; CKO: Conditional knockout; ELISA: Enzyme linked immunosorbent assay; FASN: Fatty acid synthase; GTT: Glucose tolerance test; GSDMD: Gasdermin D; HOMA-IR: Homeostatic model assessment of insulin resistance; IR: Insulin resistance; ITT: Insulin tolerance test; KD: Ketogenic diet; NLRP3: Recombinant NLR Family Pyrin Domain Containing Protein 3; PBS: Phosphate-buffered saline; PES1: Pescadillo 1; qRT-PCR: Quantitative real-time PCR; SD: Stand- ard diet; siRNA: Small interfering RNA; SREBP1c: Sterol regulatory element binding protein $1 \mathrm{C}$; SREBP2: Sterol regulatory element binding protein 2; T2DM: Type 2 diabetes mellitus; TC: Total cholesterol; TG: Triglycerides; $\beta$-HB: $\beta$-Hydroxybutyric acid.}

\section{Supplementary Information}

The online version contains supplementary material available at https://doi. org/10.1186/s10020-021-00429-6.

\footnotetext{
Additional file 1: Figure S1. Determination of cell viability. a Optimal time and concentration of $\beta-\mathrm{HB}$ treatment was determined based on the cell viability by CCK8 test, including concentration gradient $(0,0.25$, $1,2,4 \mathrm{mM})$, treatment times $(0,12,24,48 \mathrm{~h}) . \mathrm{b}, \mathrm{c}$ Protein levels of PES1 in hepatocytes were detected in different concentrations and times of $\beta-\mathrm{HB}$ treatment. Data were shown as mean \pm SEM for each experiment performed independently 3 times. ${ }^{*} P<0.05$, ${ }^{* *} P<0.01$, ${ }^{* *} P<0.001$ compared with control (ANOVA, Student-Newman-Keuls q test). Figure S2. Hepatic Pes1 gene transcription in CKO mice was detected by qRT-PCR. Data were shown as mean \pm SEM for each experiment performed independently 3 times. ${ }^{* P}<0.05,{ }^{* * P}<0.01,{ }^{* * *} P<0.001$ compared with control (Student's $\mathrm{t}$ test). Figure $\mathbf{S 3}$. KD enhanced the fatty acid $\beta$-oxidation in normal and diabetic mice. $a, b$ The protein levels of hepatic CPT1 and HMGCS2 were detected by Immunoblotting. Values are means \pm SEM for each experiment performed independently 3 times. SD (Standard diet), KD
} 
(Ketogenic diet). ${ }^{*} \mathrm{P}<0.05,{ }^{* * \mathrm{P}}<0.01,{ }^{* * *} \mathrm{P}<0.001$ compared with control for C57BL/6J, ${ }^{\#} P<0.05,{ }^{\# \#} P<0.01,{ }^{\# \# \#<<0.001 ~ c o m p a r e d ~ w i t h ~ c o n t r o l ~ f o r ~}$ $K_{K A},{ }^{+} P<0.05,{ }^{++} P<0.01,{ }^{+++} P<0.001$ compared with C57BL/6J (ANOVA, Student-Newman-Keuls q test). Figure S4. The ratios of $\beta$-HB levels in the C57BL/6J and KKAY mice fed with KD and SD. Values are means \pm SEM for each experiment performed independently 3 times. SD (Standard diet), KD (Ketogenic diet).

\section{Acknowledgements}

We would like to thank all the colleagues in our research team.

\section{Authors' contributions}

$\mathrm{KC}$ and ZJ designed the research, provide financial support and revised/edited the manuscript; $Y L$ provided partial research fund; $J Z$, JL, and $Y J$ performed all experiments; JZ collected and analyzed the data; JZ and YL wrote the draft of manuscript. All authors discussed and approved the manuscript. All authors read and approved the final manuscript.

\section{Funding}

This work is supported by the National Natural Science Foundation of China (NSFC, 81570786 to K.C, 82070986 to Z.J, 81770295 to Y.L), Wanjiang scholar grants from Anhui Province of China (9101041203 to Z.J), Outstanding young scientist program of Anhui Province (2008085J34 to Y.L).

\section{Declarations}

\section{Ethics approval and consent to participate}

The study was approved by the Institutional Animal Care and Use Committee of Anhui Medical University and performed following the relevant guidelines and regulations.

\section{Consent for publication}

Not applicable.

\section{Competing interests}

The authors declare no conflict of interest.

\section{Author details}

1 Department of Nutrition and Food Hygiene, School of Public Health, Anhui Medical University, Hefei 230032, Anhui, China. ${ }^{2}$ Department of Anesthesiology, The First Affiliated Hospital, Anhui Medical University, Hefei 230032, Anhui, China. ${ }^{3}$ Department of Health Inspection and Quarantine, School of Public Health, Anhui Medical University, Hefei 230032, Anhui, China. ${ }^{4}$ Department of Ophthalmology, The Second Affiliated Hospital, Anhui Medical University, Hefei 230021, Anhui, China.

Received: 30 September 2021 Accepted: 20 December 2021

Published online: 03 January 2022

\section{References}

Abbasi J. Interest in the ketogenic diet grows for weight loss and type 2 diabetes. JAMA. 2018:319:215-7.

Allagnat F, Fukaya M, Nogueira TC, Delaroche D, Welsh N, Marselli L, et al. C/EBP homologous protein contributes to cytokine-induced pro-inflammatory responses and apoptosis in $\beta$-cells. Cell Death Differ. 2012;19:1836-46.

Allen BG, Bhatia SK, Anderson CM, Eichenberger-Gilmore JM, Sibenaller ZA, Mapuskar KA, et al. Ketogenic diets as an adjuvant cancer therapy: history and potential mechanism. Redox Biol. 2014;2:963-70.

Allende ML, Amsterdam A, Becker T, Kawakami K, Gaiano N, Hopkins N. Insertional mutagenesis in zebrafish identifies two novel genes, pescadillo and dead eye, essential for embryonic development. Genes Dev. 1996;10:3141-55.

Anand PK. Lipids, inflammasomes, metabolism, and disease. Immunol Rev. 2020;297:108-22.
Augustin K, Khabbush A, Williams S, Eaton S, Orford M, Cross JH, et al. Mechanisms of action for the medium-chain triglyceride ketogenic diet in neurological and metabolic disorders. Lancet Neurol. 2018;17:84-93.

Badman MK, Kennedy AR, Adams AC, Pissios P, Maratos-Flier E. A very low carbohydrate ketogenic diet improves glucose tolerance in ob/ob mice independently of weight loss. Am J Physiol Endocrinol Metab. 2009:297:E1197-204.

Castellana M, Conte E, Cignarelli A, Perrini S, Giustina A, Giovanella L, et al. Efficacy and safety of very low calorie ketogenic diet (VLCKD) in patients with overweight and obesity: a systematic review and meta-analysis. Rev Endocr Metab Disord. 2020;21:5-16.

Cheng L, Li J, Han Y, Lin J, Niu C, Zhou Z, et al. PES1 promotes breast cancer by differentially regulating ERa and ERB. J Clin Invest. 2012;122:2857-70.

Cheng L, Yuan B, Ying S, Niu C, Mai H, Guan X, et al. PES1 is a critical component of telomerase assembly and regulates cellular senescence. Sci Adv. 2019;5:eaav1090.

Choi YJ, Jeon SM, Shin S. Impact of a ketogenic diet on metabolic parameters in patients with obesity or overweight and with or without type 2 diabetes: a meta-analysis of randomized controlled trials. Nutrients. 2020;12:2005

Du YC, Stillman B. Yph1p, an ORC-interacting protein: potential links between cell proliferation control, DNA replication, and ribosome biogenesis. Cell. 2002; 109:835-48

Ellenbroek JH, van Dijck L, Töns HA, Rabelink TJ, Carlotti F, Ballieux BE, et al. Long-term ketogenic diet causes glucose intolerance and reduced $\beta$ - and a-cell mass but no weight loss in mice. Am J Physiol Endocrinol Metab. 2014;306:E552-8.

Fan P, Wang B, Meng Z, Zhao J, Jin X. PES1 is transcriptionally regulated by BRD4 and promotes cell proliferation and glycolysis in hepatocellular carcinoma. Int J Biochem Cell Biol. 2018;104:1-8.

Gentile CL, Weir TL. The gut microbiota at the intersection of diet and human health. Science. 2018;362:776-80.

Guo Y, Zhang C, Shang FF, Luo M, You Y, Zhai Q, et al. Ketogenic diet ameliorates cardiac dysfunction via balancing mitochondrial dynamics and inhibiting apoptosis in type 2 diabetic mice. Aging Dis. 2020;11:229-40.

Holdt LM, Stahringer A, Sass K, Pichler G, Kulak NA, Wilfert W, et al. Circular non-coding RNA ANRIL modulates ribosomal RNA maturation and atherosclerosis in humans. Nat Commun. 2016:7:12429.

Klil-Drori AJ, Azoulay L, Pollak MN. Cancer, obesity, diabetes, and antidiabetic drugs: is the fog clearing? Nat Rev Clin Oncol. 2017;14(2):85-99.

Landry MJ, Crimarco A, Perelman D, Durand LR, Petlura C, Aronica L, et al. Adherence to ketogenic and Mediterranean study diets in a crossover trial: the Keto-Med randomized trial. Nutrients. 2021;13(3):967.

Li Z, Heber D. Ketogenic diets. JAMA. 2020;323:386.

Li J, Zhuang Q, Lan X, Zeng G, Jiang X, Huang Z. PES1 differentially regulates the expression of ERa and ERB in ovarian cancer. IUBMB Life. 2013;65:1017-25.

Li J, Huang Q, Long X, Zhang J, Huang X, Aa J, et al. CD147 reprograms fatty acid metabolism in hepatocellular carcinoma cells through Akt/mTOR/ SREBP1C and P38/PPARa pathways. J Hepatol. 2015;63:1378-89.

Liu R, Li X, Huang Z, Zhao D, Ganesh BS, Lai G, et al. C/EBP homologous protein-induced loss of intestinal epithelial stemness contributes to bile duct ligation-induced cholestatic liver injury in mice. Hepatology. 2018;67:1441-57

Magliano DJ, Sacre JW, Harding JL, Gregg EW, Zimmet PZ, Shaw JE. Youngonset type 2 diabetes mellitus — implications for morbidity and mortality. Nat Rev Endocrinol. 2020;16:321-31.

Maris M, Overbergh L, Gysemans C, Waget A, Cardozo AK, Verdrengh E, et al. Deletion of C/EBP homologous protein (Chop) in C57BL/6 mice dissociates obesity from insulin resistance. Diabetologia. 2012;55:1167-78.

McGuire DK, Van de Werf F, Armstrong PW, Standl E, Koglin J, Green JB, et al. Association between Sitagliptin use and heart failure hospitalization and related outcomes in type 2 diabetes mellitus: secondary analysis of a randomized clinical trial. JAMA Cardiol. 2016:1:126-35.

Newman JC, Verdin E. $\beta$-hydroxybutyrate: a signaling metabolite. Annu Rev Nutr. 2017:37:51-76.

Offermanns S. Hydroxy-carboxylic acid receptor actions in metabolism. Trends Endocrinol Metab. 2017:28:227-36.

Ponugoti B, Kim DH, Xiao Z, Smith Z, Miao J, Zang M, et al. SIRT1 deacetylates and inhibits SREBP-1C activity in regulation of hepatic lipid metabolism. $J$ Biol Chem. 2010;285:33959-70. 
Poplawski MM, Mastaitis JW, Isoda F, Grosjean F, Zheng F, Mobbs CV. Reversal of diabetic nephropathy by a ketogenic diet. PLOS ONE. 2011;6:e18604.

Puchalska P, Crawford PA. Multi-dimensional roles of ketone bodies in fuel metabolism, signaling, and therapeutics. Cell Metab. 2017;25:262-84.

Shimazu T, Hirschey MD, Hua L, Dittenhafer-Reed KE, Schwer B, Lombard $\mathrm{DB}$, et al. SIRT3 deacetylates mitochondrial 3-hydroxy-3-methylglutaryl CoA synthase 2 and regulates ketone body production. Cell Metab. 2010;12:654-61.

Shlomai G, Neel B, LeRoith D, Gallagher EJ. Type 2 diabetes mellitus and cancer: the role of pharmacotherapy. J Clin Oncol. 2016;34:4261-9.

Stienstra R, van Diepen JA, Tack CJ, Zaki MH, van de Veerdonk FL, Perera D, et al. Inflammasome is a central player in the induction of obesity and insulin resistance. Proc Natl Acad Sci USA. 2011;108:15324-9.

Thio LL, Erbayat-Altay E, Rensing N, Yamada KA. Leptin contributes to slower weight gain in juvenile rodents on a ketogenic diet. Pediatr Res. 2006;60:413-7.

Todoric J, Di Caro G, Reibe S, Henstridge DC, Green CR, Vrbanac A, et al. Fructose stimulated de novo lipogenesis is promoted by inflammation. Nat Metab. 2020;2:1034-45.

Wang Y, Viscarra J, Kim SJ, Sul HS. Transcriptional regulation of hepatic lipogenesis. Nat Rev Mol Cell Biol. 2015;16:678-89.

Watanabe M, Tozzi R, Risi R, Tuccinardi D, Mariani S, Basciani S, et al. Beneficial effects of the ketogenic diet on nonalcoholic fatty liver disease: a comprehensive review of the literature. Obes Rev. 2020;21:e13024.

Xu L, Li Y, Yin L, Qi Y, Sun H, Sun P, et al. miR-125a-5p ameliorates hepatic glycolipid metabolism disorder in type 2 diabetes mellitus through targeting of STAT3. Theranostics. 2018;8:5593-609.

Yancy WS Jr, Mitchell NS, Westman EC. Westman ketogenic diet for obesity and diabetes. JAMA Intern Med. 2019;179:1734-5.

Yang Z, Mi J, Wang Y, Xue L, Liu J, Fan M, et al. Effects of low-carbohydrate diet and ketogenic diet on glucose and lipid metabolism in type 2 diabetic mice. Nutrition. 2021;89:111230.

Youm YH, Nguyen KY, Grant RW, Goldberg EL, Bodogai M, Kim D, et al. The ketone metabolite $\beta$-hydroxybutyrate blocks NLRP3 inflammasomemediated inflammatory disease. Nat Med. 2015;21:263-9.

Yu Y, Wang F, Wang J, Zhang D, Zhao X. Ketogenic diet attenuates aging-associated myocardial remodeling and dysfunction in mice. Exp Gerontol. 2020;140:111058

Zhang X, Qin J, Zhao Y, Shi J, Lan R, Gan Y, et al. Long-term ketogenic diet contributes to glycemic control but promotes lipid accumulation and hepatic steatosis in type 2 diabetic mice. Nutr Res. 2016;36:349-58.

Zhang J, Zhang Y, Yang X, Wang J, Xu Y, Wang R, et al. Diabetic bladder dysfunction in T2D KK-Ay mice and its changes in the level of relevant gene expression. Biomed Pharmacother. 2020;131:110706.

Zheng Y, Ley SH, Hu FB. Global aetiology and epidemiology of type 2 diabetes mellitus and its complications. Nat Rev Endocrinol. 2018;14:88-98.

\section{Publisher's Note}

Springer Nature remains neutral with regard to jurisdictional claims in published maps and institutional affiliations.

Ready to submit your research? Choose BMC and benefit from:

- fast, convenient online submission

- thorough peer review by experienced researchers in your field

- rapid publication on acceptance

- support for research data, including large and complex data types

- gold Open Access which fosters wider collaboration and increased citations

- maximum visibility for your research: over $100 \mathrm{M}$ website views per year

At BMC, research is always in progress.

Learn more biomedcentral.com/submissions 